\title{
Modeling of Output Characteristics of a UV Cu+ Ne-CuBr Laser
}

\section{Snezhana Georgieva Gocheva-Ilieva, ${ }^{1}$ Desislava Stoyanova Voynikova, ${ }^{1}$ and Iliycho Petkov Iliev ${ }^{2}$}

${ }^{1}$ Department of Applied Mathematics and Modeling, University of Plovdiv, 24 Tzar Assen Street, 4000 Plovdiv, Bulgaria

${ }^{2}$ Department of Physics, Technical University of Sofia, Branch Plovdiv, 25 Tzanko Djusstabanov Street, 4000 Plovdiv, Bulgaria

Correspondence should be addressed to

Snezhana Georgieva Gocheva-Ilieva, snegocheva@yahoo.com

Received 2 March 2012; Accepted 12 May 2012

Academic Editor: Hung Nguyen-Xuan

Copyright (c) 2012 Snezhana Georgieva Gocheva-Ilieva et al. This is an open access article distributed under the Creative Commons Attribution License, which permits unrestricted use, distribution, and reproduction in any medium, provided the original work is properly cited.

This paper examines experiment data for a Ne-CuBr UV copper ion laser excited by longitudinal pulsed discharge emitting in multiline regime. The flexible multivariate adaptive regression splines (MARSs) method has been used to develop nonparametric regression models describing the laser output power and service life of the devices. The models have been constructed as explicit functions of 9 basic input laser characteristics. The obtained models account for local nonlinearities of the relationships within the various multivariate subregions. The built best MARS models account for over $98 \%$ of data. The models are used to estimate the investigated output laser characteristics of existing UV lasers. The capabilities for using the models in predicting existing and future experiments have been demonstrated. Specific analyses have been presented comparing the models with actual experiments. The obtained results are applicable for guiding and planning the engineering experiment. The modeling methodology can be applied for a wide range of similar lasers and laser devices.

\section{Introduction}

During the process of engineering design, together with the purely experimental investigations, various methods for mathematical modeling are also applied. Standard mathematical models usually include differential and integral equations, optimization, or other types of mathematical problems through which the processes occurring in the laser medium are described and the behavior of the system is explored by simulating its various states [15]. The disadvantage of these methods is the difficulty in obtaining an explicit estimate of 
the final output characteristics such as laser output power, overall efficiency, or service life of the device. If experiment data from the process of developing laboratory and industrial prototypes is available, another approach is the application of statistical methods. These are useful in finding basic relationships within data for the specific type of device and the utilization of obtained results for guiding and planning of the engineering experiment. In the field of metal vapor lasers, traditional parametric statistical methods have been used in [69]. In [6, 9], MARS models are applied to different relationships between the data for $\mathrm{CuBr}$ lasers in the visible range. For ultraviolet lasers with copper bromide vapors, some particular MARS models for examining laser output power have been developed in [6, 10].

This paper conducts a statistical investigation of laser generation and service life of UV copper bromide vapor lasers. This new type of laser source is currently being developed at the Laboratory of Metal Vapor Lasers, Bulgarian Academy of Sciences [11-13]. The main experiment results obtained so far, used in this paper, have been published in [14-20].

The goal of this study is to determine the relationships between 9 input laser characteristics and two output characteristics: laser generation and laser service life by applying the MARS method based on available experiment data. The following problems are solved: (1) determining which input quantities (supplied power, geometric design of the tube, neon pressure, reservoirs temperature, etc.) influence significantly the change of the considered output characteristics; (2) defining adequate regression models describing explicitly the relationship between independent variables and dependent quantities; (3) application of the models for estimating known experiments; (4) application of the models for predicting experiments, in particular for guiding design and construction work when developing new UV copper bromide vapor lasers; (5) using the models to investigate the local influence of the main input laser quantities on output ones.

The examination has been conducted using MARS statistical software [21, 22].

\section{Subject of Study}

We investigate experiment data for an ultraviolet copper ion excited copper bromide vapor laser ( $\mathrm{UV} \mathrm{Cu}+\mathrm{Ne}-\mathrm{CuBr}$ laser). This ultraviolet laser is a promising innovative product with unique characteristics and stable operation. The first lasers of this type were constructed by the Laboratory of MVLs at the Institute of Solid State Physics of Bulgarian Academy of Sciences in 1999 [11, 14]. It is characterized by high quality of the laser beam and high output power. During the last decade, this type of laser has been the subject of intense experimental studies and its characteristics have been significantly improved (see $[19,20]$ and the cited therein detailed literature with experimental results).

Ultraviolet laser generation can be achieved using copper or gold ions. The typical technological problems of copper ion lasers-copper deposits on the inside walls of the tube and high discharge temperature-were solved thanks to the development of a new UV Ne-CuBr laser with a nanosecond pulsed longitudinal discharge. This laser emits in the deep ultraviolet spectrum on five spectral lines -248.6, 252.9, 259.7, 260.0, and $270.3 \mathrm{~nm}$. Experimentally, a record average output power of $1.3 \mathrm{~W}$ has been obtained at all five lines and $0.85 \mathrm{~W}$ at the $248.6 \mathrm{~nm}$ line, but the laser emission was achieved only for a period of 20 hours, due to the technological problems $[15,16]$. It has also been determined that adding small amounts of hydrogen (0.02-0.04 Torr) results in a twofold increase of laser generation [19]. Further improvement by overcoming this problem was reported in [20].

This laser has large fields of application including medicine, microelectronics, photolithography, genetic engineering, and scientific research [23-25]. Due to the narrow 
Table 1: Technical characteristics of a UV copper ion vapor laser [20].

\begin{tabular}{lc}
\hline Characteristic & Values \\
\hline Radiation wavelength & $248.6,252.9,259.1,260.0,270.3 \mathrm{~nm}$ \\
Operating mode & Periodic pulse, self-heating \\
Pulse rate frequency & $15-25 \mathrm{kHz}$ \\
Pulse length & $20-50 \mathrm{~ns}$ \\
Input electrical power & $1-2.5 \mathrm{~kW}$ \\
Average output power & $0.2-1.3 \mathrm{~W}$ \\
Laser efficiency & $<1 \%$ \\
Total service life & $\mathrm{Up} \mathrm{to} 1000 \mathrm{hours}$ \\
Active medium temperature & $1000 \mathrm{~K}$ \\
Start time & $15-20 \mathrm{~min}$ \\
Buffer gas Neon & $7-30 \mathrm{Torr}$ \\
Hydrogen admixture & $0-0.06 \mathrm{Torr}$ \\
Active zone length & $60-100 \mathrm{~cm}$ \\
Inside tube diameter & $4-26 \mathrm{~mm}$ \\
\hline
\end{tabular}

emission range of just a few spectral lines and the high coherence of the beam, it is used for processing, which requires high resolution, such as recording information, fluorescence, high-precision drilling, cutting, cleaning, modification of newly developed materials, and so forth. Technological applications of the UV lasers created at the Laboratory of Metal Vapor Lasers are achieved. Laser-induced modification by the use of UV laser radiation with $248.6 \mathrm{~nm}$ wavelength has been performed in a conducting polymer. A significant growth in the electrocrystallization of copper on polymer layers was obtained. The refractivity of polymer layers in ultraviolet spectral regions was determined. Micronic holes of 10, 20, and 40 microns in size were drilled in glass, polymer, and ZnSe [20].

The schematic of the ultraviolet Ne-CuBr laser tube is shown in Figure 1. Its main technical characteristics are given in Table 1.

The goal of subsequent development of the examined UV lasers is to investigate the possibilities for improving its output characteristics: output power and service life.

\section{Data Description}

A total of 9 laser characteristics (independent variables, predictors) are studied, which are of physical significance and are considered independent. The variables we will examine are as follows: $D, \mathrm{~mm}$ : inside diameter of the laser tube; $\mathrm{DR}, \mathrm{mm}$ : inside diameter of the internal rings in the ceramic tube or quartz tube insert; $L, \mathrm{~cm}$ : length of the active zone (distance between the electrodes); $P_{\mathrm{IN} 2}, \mathrm{~kW}$ : electric power supplied to the active volume, taking into account $50 \%$ losses of the total supplied power $P_{\mathrm{IN}}$; $P_{\mathrm{NE}}$, Torr: neon gas pressure; $P_{\mathrm{RF}}, \mathrm{kHz}$ : pulse rate frequency; $P_{\mathrm{H} 2}$, Torr: hydrogen gas pressure; $C, \mathrm{pF}$ : equivalent capacity of the condensation battery; $\mathrm{TR},{ }^{\circ} \mathrm{C}$ : temperature of the copper bromide reservoir (equal to the temperature of the outer side of the quartz tube).

The dependent variables are laser output power (laser generation) $P_{\text {out }}, \mathrm{W}$, and laser service life $L_{\text {Time, }}$ hours. 


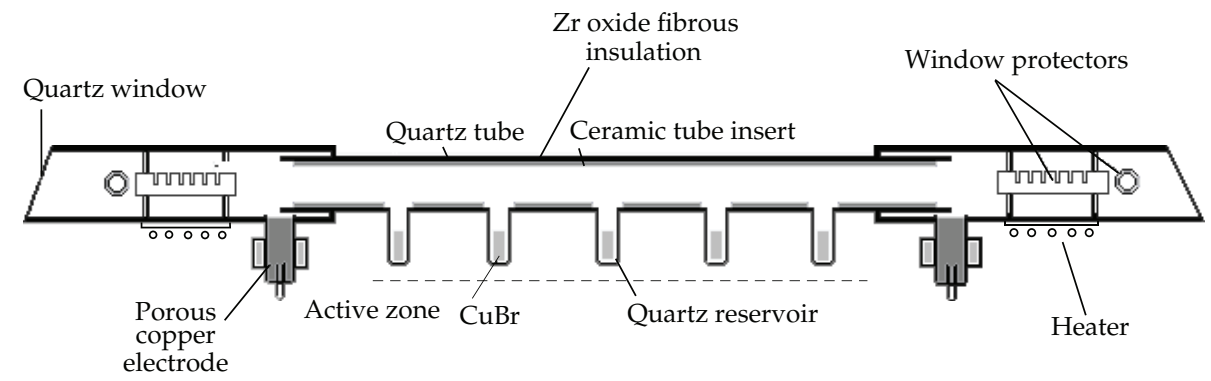

Figure 1: Longitudinal principle scheme of the laser tube of a UV copper ion laser.

This study uses the data from $n=238$ experiments for 11 different laser devices, reported in [11-20]. The scatter plot of the relationships for each two of the eleven variables (9 independent and 2 dependent) is given in Figure 2.

The descriptive properties of the data indicate that applying the standard (conventional) parametric methods is not recommendable as the basic conditions for their validity are violated [6]. More specifically, the two dependent variables are not normally distributed. There is also certain collinearity between independent variables. For that reason, in this paper, nonparametric statistical techniques are used, choosing in this case the MARS method.

\section{MARS Method}

The relatively new nonparametric MARS technique that performs well with complex data structures has been applied [21, 22, 26]. MARS allows building flexible models capable of describing local relationships in a multidimensional region of data, within which subregions are defined. For each of these multidimensional subregions, the most suitable type of relationship is chosen and a general model is built, which approximates as closely as possible the actual data in accordance with a specific criterion.

Let us denote the independent variables by $X=\left(x_{1}, x_{2}, \ldots, x_{p}\right)$ and their dependent one by $y=y(X)$. We assume that $y, x_{1}, x_{2}, \ldots, x_{p}$ are vectors with dimension $n$. For our data $n=238, p=9$. The general form of a MARS model $\hat{y}_{[M]}$ of the data for $y$ has the form

$$
\widehat{y}_{[M]}=b_{0}+\sum_{j=1}^{M} b_{j} \mathrm{BF}_{j}(\mathrm{X})
$$

where $b_{0}, b_{j}, j=1,2, \ldots, M$ are the sought constant coefficients of the model, $M$ the number of functions included in the model, and $\mathrm{BF}_{j}(X)$ basis functions of the selected type.

In the linear case, basis functions are presented in the form of one of the following two one-dimensional "mirror" functions:

$$
\mathrm{BF}_{j}(X)=\max \left(0, x_{k}-c_{k}\right) \quad \text { or } \mathrm{BF}_{j}(X)=\max \left(0, c_{k}-x_{k}\right)
$$

Here $c_{k}$ is a constant, called a knot of the basis function with values in the definition interval of the predictor variable $x_{k}$. In the linear case, model (4.1) presented as a sum of basis functions of type (4.2) is plotted graphically in the form of a piecewise linear function. 




Figure 2: Scatter plot of all investigated data of UV Cu+ $\mathrm{Ne}-\mathrm{CuBr}$ vapor lasers.

When building nonlinear MARS models, basis functions $\mathrm{BF}_{j}(X)$ can also include the products of two or more functions of the form (4.2) without repeating the index $k$. The maximum number of multipliers is called order of interaction. The linear model has an order of interaction 1.

We have to note that unlike ordinary interpolation splines, in the MARS model (4.1), the knots of the basis functions, their number $M$, the selection of functions, and the subintervals of determination are not known in advance. These are determined by different optimization conditions and prerequisites for estimating the proximity of the model to real data on $y(X)$.

When building the models, the researcher sets an initial maximum number $M_{0}$ of basis functions and the maximum order of interaction. The recommendation is $M_{0} \geq 3 p$ [21]. MARS procedures systematically select subintervals and subregions for each predictor, estimate the degree of significance to the model of a given basis function, and exclude those that do not contribute to the improvement of the model. Several different criteria are used to evaluate the model after each step. One criterion is the minimization of the sum of squares of errors:

$$
\operatorname{SSE}_{[Q]}=\sum_{i=1}^{n}\left(y_{i}-\widehat{y}_{[Q]}\left(X_{i}\right)\right)^{2}
$$

for a model with a $Q$ number of current basis functions. Another basic criterion is the minimization of the coefficient of general cross-validation (GCV) [19, 20]. According to 
Table 2: Main statistics of the constructed best MARS models of laser output power $P_{\text {out }}$ of UV copper ion lasers.

\begin{tabular}{|c|c|c|c|c|c|}
\hline Model & $R^{2}$ & MARS GCV $R^{2}$ & Sum of Squares & $\mathrm{BF}$ in model & Predictors \\
\hline$(30 ; 1)$ & 0.921 & 0.894 & 97.18 & 13 & $P_{\mathrm{NE}}, P_{\mathrm{IN} 2}, L, P_{\mathrm{H} 2}, P_{\mathrm{RF}}, D, C, \mathrm{DR}$ \\
\hline$(40 ; 1)$ & 0.921 & 0.892 & 97.18 & 13 & $P_{\mathrm{NE}}, P_{\mathrm{IN} 2}, L, P_{\mathrm{H} 2}, P_{\mathrm{RF}}, D, C, \mathrm{DR}$ \\
\hline$(50 ; 1)$ & 0.922 & 0.890 & 96.83 & 14 & $P_{N E}, P_{\mathrm{IN} 2}, L, P_{\mathrm{H} 2}, P_{\mathrm{RF}}, D, C, \mathrm{DR}$ \\
\hline$(30 ; 2)$ & 0.968 & 0.940 & 63.37 & 23 & $P_{\mathrm{IN} 2}, P_{\mathrm{NE}}, P_{\mathrm{H} 2}, \mathrm{DR}, L, P_{\mathrm{RF}}, C$ \\
\hline$(35 ; 2)$ & 0.977 & 0.951 & 55.80 & 27 & $P_{\mathrm{IN} 2}, P_{\mathrm{NE}}, P_{\mathrm{H} 2}, \mathrm{DR}, L, P_{\mathrm{RF}}, C$ \\
\hline$(40 ; 2)$ & 0.980 & 0.955 & 50.34 & 28 & $P_{\mathrm{IN} 2}, P_{\mathrm{NE}}, P_{\mathrm{H} 2}, \mathrm{DR}, L, P_{\mathrm{RF}}, C, D$ \\
\hline$(45 ; 2)$ & 0.982 & 0.959 & 47.67 & 28 & $P_{\mathrm{IN} 2}, P_{\mathrm{NE}}, P_{\mathrm{H} 2}, \mathrm{DR}, L, P_{\mathrm{RF}}, C, D$ \\
\hline$(50 ; 2)$ & 0.984 & 0.957 & 45.50 & 31 & $P_{\mathrm{IN} 2}, P_{\mathrm{NE}}, P_{\mathrm{H} 2}, \mathrm{DR}, L, P_{\mathrm{RF}}, C, D$ \\
\hline$(30 ; 3)$ & 0.978 & 0.957 & 52.82 & 23 & $P_{\mathrm{IN} 2}, \mathrm{DR}, P_{\mathrm{NE}}, L, P_{\mathrm{H} 2}, \mathrm{TR}, P_{\mathrm{RF}}, D, C$ \\
\hline$(35 ; 3)$ & 0.981 & 0.959 & 49.60 & 26 & $P_{\mathrm{IN} 2}, \mathrm{DR}, P_{\mathrm{NE}}, L, P_{\mathrm{H} 2}, \mathrm{TR}, P_{\mathrm{RF}}, D, C$ \\
\hline$(40 ; 3)$ & 0.984 & 0.965 & 44.92 & 27 & $P_{\mathrm{IN} 2}, \mathrm{DR}, P_{\mathrm{NE}}, L, P_{\mathrm{H} 2}, P_{\mathrm{RF}}, D, C, \mathrm{TR}$ \\
\hline$(45 ; 3)$ & 0.984 & 0.964 & 44.92 & 27 & $P_{\mathrm{IN} 2}, \mathrm{DR}, P_{\mathrm{NE}}, L, P_{\mathrm{H} 2}, \mathrm{P}_{\mathrm{RF}}, D, C, \mathrm{TR}$ \\
\hline$(50 ; 3)$ & 0.985 & 0.965 & 44.42 & 27 & $P_{\mathrm{IN} 2}, \mathrm{DR}, P_{\mathrm{NE}}, \mathrm{L}, P_{\mathrm{H} 2}, \mathrm{P}_{\mathrm{RF}}, D, C, \mathrm{TR}$ \\
\hline
\end{tabular}

which "the best" model of $M_{1}$ models with $m$ basis functions is the one that minimizes the expression

$$
\operatorname{GCV}_{[m]}=\frac{\sum_{i=1}^{n}\left(y_{i}-\widehat{y}_{[m]}\left(X_{i}\right)\right)^{2}}{n(1-C(m) / n)^{2}}, \quad m=1,2, \ldots, M_{1}
$$

where $C(m)=m+\delta(m-1) / 2, \delta \in[2,3], X_{i}=\left(x_{1 i}, x_{2 i}, \ldots, x_{p i}\right), y_{i}=y\left(x_{1 i}, x_{2 i}, \ldots, x_{p i}\right)$.

As a whole, the implementation of MARS method algorithms allows the automatic definition of those predictors that influence the examined dependent variable, as well as the degree of this influence, removing the statistically insignificant predictors. The final model usually requires that the residuals of the model are normally distributed.

An advantage of the method is that the resulting models are simpler in form and can easily be interpreted during their practical application.

Within this study only the best MARS models are presented. All nine variables are initially introduced as predictors. We use the notation $\left(M_{0}, r\right)$ for a model with $M_{0}$ initial maximum number of allowed basis functions (BFs) and up to $r$ th order of interactions, $r \geq 1$.

\section{Building MARS Models of the Laser Generation of an UV Laser}

First, we will present the best MARS models of laser generation $P_{\text {out }}$.

The basic statistic figures of the constructed models are given in Table 2. We will describe in more detail two of these models: the best piecewise linear model with no interactions and the best model with first-order interactions.

\subsection{Linear MARS Models of $P_{\text {out }}$}

Out of the linear models, we will present model $(40 ; 1)$. The figures of other linear models are given in the upper part of Table 2. 
The generated best MARS model (40;1) includes the following 13 basis functions (BFs) with 8 predictors:

$$
\begin{aligned}
\mathrm{BF} 1 & =\max (0, \mathrm{DR}-5.7), \\
\mathrm{BF} 3 & =\max \left(0, P_{\mathrm{IN} 2}-525\right), \\
\mathrm{BF} 4 & =\max \left(0, P_{\mathrm{H} 2}-0.03\right), \\
\mathrm{BF} 5 & =\max \left(0,0.03-P_{\mathrm{H} 2}\right), \\
\mathrm{BF} 8 & =\max \left(0, P_{\mathrm{RF}}-19.5\right), \\
\mathrm{BF} 9 & =\max (0, L-90), \\
\mathrm{BF} 11 & =\max (0, C-372.184), \\
\mathrm{B} 12 & =\max (0, D-9.5), \\
\mathrm{BF} 14 & =\max (0, L-86.5), \\
\mathrm{BF} 16 & =\max \left(0, P_{\mathrm{NE}}-19.3\right), \\
\mathrm{BF} 18 & =\max \left(0, P_{\mathrm{NE}}-18.6\right), \\
\mathrm{B} 20 & =\max \left(0, P_{\mathrm{NE}}-20\right), \\
\mathrm{B} 26 & =\max (0, D-7.1) .
\end{aligned}
$$

The obtained regression model for laser output power $P_{\text {out }}$ with these functions is

$$
\begin{aligned}
\widehat{P}_{\text {out }}= & 403.83+49.71 \mathrm{BF} 1+0.901119 \mathrm{BF} 3-11325.2 \mathrm{BF} 4-9840.28 \mathrm{BF} 5 \\
& +23.5177 \mathrm{BF} 8-79.7689 \mathrm{BF} 9+0.453381 \mathrm{BF} 11+218.763 \mathrm{BF} 12 \\
& +42.4055 \mathrm{BF} 14-1007.34 \mathrm{BF} 16+514.971 \mathrm{BF} 18+480.904 \mathrm{BF} 20 \\
& -215.734 \mathrm{BF} 26 .
\end{aligned}
$$

The model (5.1)-(5.2) can easily be used to estimate or predict laser generation. For example, let us take case $i=128$, with output laser power $P_{\text {out }_{128}}=900 \mathrm{~mW}$ [20] at the following values of the independent variables:

$$
\begin{aligned}
X_{128}= & \left(D=7.1 \mathrm{~mm}, \mathrm{DR}=7.1 \mathrm{~mm}, L=86.5 \mathrm{~cm}, P_{\mathrm{IN}}=1500 \mathrm{~W}, P_{\mathrm{NE}}=16.7 \text { Torr },\right. \\
& \left.P_{\mathrm{H} 2}=0.03 \text { Torr }, P_{\mathrm{RF}}=19.5 \mathrm{kHz}, \mathrm{C}=735 \mathrm{pF}, \mathrm{Tr}=495^{\circ} \mathrm{C}\right) .
\end{aligned}
$$

We calculate each of the functions in (5.1) consecutively. We have BF1 $=1.4$, BF3 = $1500 / 2-525=225, \mathrm{BF} 4=\mathrm{BF} 5=\mathrm{BF} 8=\mathrm{BF} 9=0$, and so forth. We substitute in model (5.2) obtaining the estimate

$$
\widehat{P}_{\text {out }_{128}}=840 \mathrm{~mW} .
$$


The absolute error in case $i=128$ is about $60 \mathrm{~mW}$, and the relative one is $7 \%$. The overall relative error for the model is $8-9 \%$, which is a satisfactory figure.

Model (5.1)-(5.2) is the best MARS model of a given type, which is selected so as to allow no overfitting of the model, as well as by using the measures of data fit SSE and GCV. The obtained basic statistics are given in Table 2. The model is significant at level 0.000.

The relative influence of individual predictors in model (5.2) is given in Table 3, column 2. It is apparent that the most significant variable is $P_{\mathrm{H} 2}$, whose influence is measured as $100 \%$, and the influence of the others is calculated against it.

The local behavior of the main predictors and their contribution to model (5.1)-(5.2) are shown in Figures 3(a)-3(h) in pure ordinal units.

\subsection{Nonlinear MARS Models}

Of the second-order models we present the best model with up to $40 \mathrm{BF}$, which is denoted as model (40; 2 ). The obtained model includes the following 28 BFs:

$$
\begin{gathered}
\mathrm{BF} 1=\max (0, \mathrm{DR}-5.7), \\
\mathrm{BF} 2=\max (0,5.7-\mathrm{DR}), \\
\mathrm{BF} 3=\max \left(0, P_{\mathrm{IN} 2}-525\right), \\
\mathrm{BF} 4=\max \left(0, P_{\mathrm{H} 2}-0.03\right), \\
\mathrm{BF} 5=\max \left(0,0.03-P_{\mathrm{H} 2}\right), \\
\mathrm{BF} 8=\max \left(0, P_{\mathrm{NE}}-19.3\right) \mathrm{BF} 3, \\
\mathrm{BF} 12=\max \left(0, P_{\mathrm{NE}}-20\right) \mathrm{BF} 3, \\
\mathrm{BF} 14=\max \left(0, P_{\mathrm{RF}}-20\right) \mathrm{BF} 3, \\
\mathrm{BF} 15=\max \left(0,20-P_{\mathrm{RF}}\right) \mathrm{BF} 3, \\
\mathrm{BF} 16=\max (0, L-90) \mathrm{BF} 3, \\
\mathrm{BF} 17=\max (0,90-L) \mathrm{BF} 3, \\
\mathrm{BF} 18=\max \left(0, P_{\mathrm{IN} 2}-650\right) \mathrm{BF} 5, \\
\mathrm{BF} 19=\max \left(0,650-P_{\mathrm{IN} 2}\right) \mathrm{BF} 5, \\
\mathrm{BF} 21=\max \left(0,21-P_{\mathrm{RF}}\right) \mathrm{BF} 5, \\
\mathrm{BF} 22=\max \left(0, P_{\mathrm{NE}}-13.5\right) \mathrm{BF} 1, \\
\mathrm{BF} 23=\max \left(0,13.5-P_{\mathrm{NE}}\right) \mathrm{BF} 1, \\
\mathrm{BF} 24=\max (0, C-372.184) \mathrm{BF} 1, \\
\mathrm{BF} 25=\max (0, L-90) \mathrm{BF} 1, \\
\mathrm{~B} 28=\max \left(0, P_{\mathrm{IN} 2}-670\right) \mathrm{BF} 1, \\
\end{gathered}
$$




$$
\begin{gathered}
\mathrm{BF} 29=\max \left(0, P_{\mathrm{NE}}-18.3\right) \mathrm{BF} 3, \\
\mathrm{BF} 31=\max \left(0, P_{\mathrm{NE}}-19.75\right) \mathrm{BF} 3, \\
\mathrm{BF} 33=\max \left(0, P_{\mathrm{NE}}-17.5\right), \\
\mathrm{BF} 34=\max \left(0,17.5-P_{\mathrm{NE}}\right), \\
\mathrm{BF} 35=\max \left(0, P_{\mathrm{NE}}-5.5\right) \mathrm{BF} 2, \\
\mathrm{BF} 36=\max (0, D-4) \mathrm{BF} 2, \\
\mathrm{BF} 37=\max \left(0, P_{\mathrm{NE}}-21.5\right) \mathrm{BF} 5, \\
\mathrm{BF} 39=\max \left(0, P_{\mathrm{NE}}-20\right) .
\end{gathered}
$$

The corresponding regression model for laser output power $P_{\text {out }}$ includes all of these functions with 8 independent variables. It has the following form:

$$
\begin{aligned}
\widehat{P}_{\text {out }}= & 687.002-5793.51 \mathrm{BF} 2+1.48468 \mathrm{BF} 3-10671 \mathrm{BF} 4-15811.5 \mathrm{BF} 5 \\
& -3.04711 \mathrm{BF} 8+0.957235 \mathrm{BF} 10+4.28622 \mathrm{BF} 12+0.164663 \mathrm{BF} 14 \\
& -1.38904 \mathrm{BF} 15-0.110126 \mathrm{BF} 16-0.0553432 \mathrm{BF} 17-59.017 \mathrm{BF} 18 \\
& +245.219 \mathrm{BF} 19+3593.7 \mathrm{BF} 21-26.8772 \mathrm{BF} 22+7.20284 \mathrm{BF} 23 \\
& +0.348349 \mathrm{BF} 24+22.0488 \mathrm{BF} 25+0.266576 \mathrm{BF} 27-0.814258 \mathrm{BF} 28 \\
& +1.31689 \mathrm{BF} 29-3.51103 \mathrm{BF} 31-52.8455 \mathrm{BF} 33-63.9875 \mathrm{BF} 34 \\
& +368.813 \mathrm{BF} 35+537.251 \mathrm{BF} 36-12825.2 \mathrm{BF} 37-180.136 \mathrm{BF} 39 .
\end{aligned}
$$

Likewise, model (5.5)-(5.6) can be used to estimate and predict output power. For example, in the same case $i=128$, with the data (5.3), we obtain the estimate

$$
\widehat{P}_{\text {out }_{128}}=856.4 \mathrm{~mW} \text {. }
$$

The absolute error in case $i=128$ is about $44 \mathrm{~mW}$, and the relative one is $5 \%$.

A part of the partial contributions of pairs of predictors in model (5.5)-(5.6) to the value of $P_{\text {out }}$ are presented in Figures 3(a)-3(d). The biggest contribution of more than 6000 units is made by the interaction between $P_{\mathrm{NE}}$ and $P_{\mathrm{H} 2}$, which reaches the highest value in a large 2D region. Predictors $C$ and DR provide almost the same contribution. The other two interactions also have given effects. 




(a)

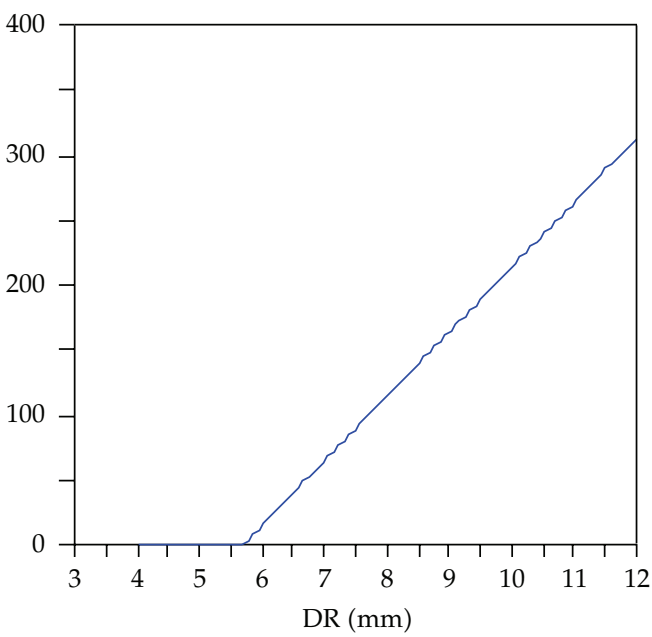

(c)



(e)

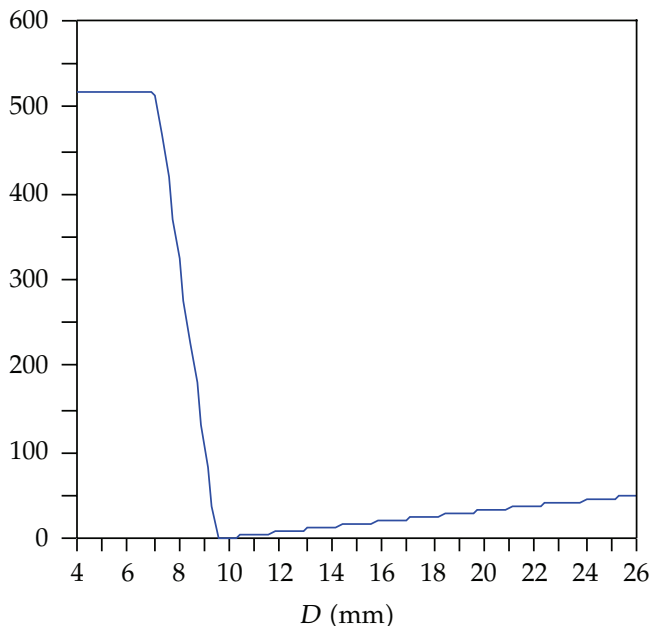

(b)



(d)

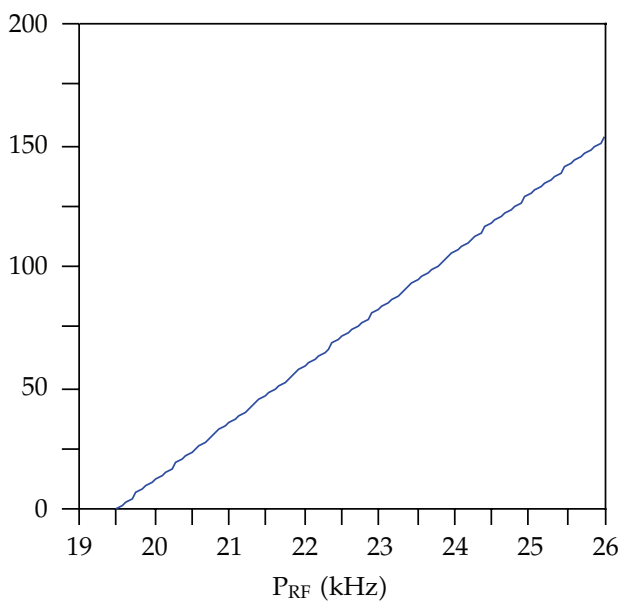

(f)

Figure 3: Continued. 


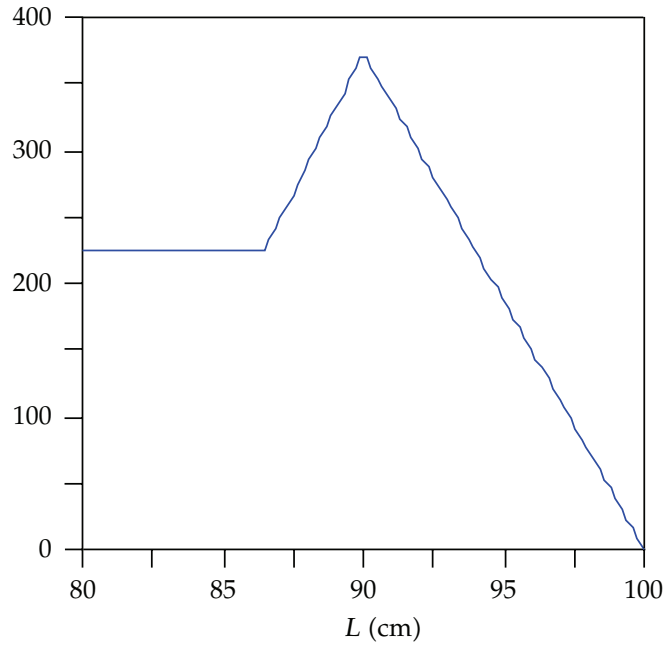

(g)

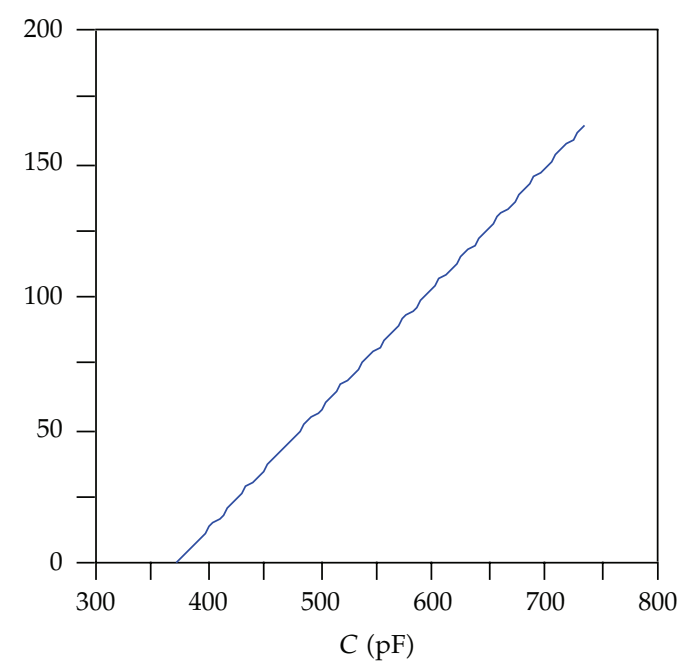

(h)

Figure 3: Graphs of the main predictors in model (5.1)-(5.2) showing their relationship with the response $P_{\text {out. }}$.

Table 3: Relative variable importance in the considered MARS model (40;1), described by (5.1)-(5.2), and model (40;2), described by (5.5)-(5.6), for laser output power $P_{\text {out }}$ of UV copper ion laser.

\begin{tabular}{lcc}
\hline Variable & In model $(40 ; 1), \%$ & In model $(40 ; 2) ; \%$ \\
\hline$P_{\mathrm{IN} 2}$ & $97.3 \%$ & $100 \%$ \\
$P_{\mathrm{NE}}$ & $100 \%$ & $68.6 \%$ \\
$P_{\mathrm{H} 2}$ & $66.8 \%$ & $59.7 \%$ \\
$\mathrm{DR}$ & $19.3 \%$ & $40.4 \%$ \\
$L$ & $76.7 \%$ & $35.4 \%$ \\
$P_{\mathrm{RF}}$ & $55.2 \%$ & $28.3 \%$ \\
$C$ & $30.9 \%$ & $22.5 \%$ \\
$D$ & $53.0 \%$ & $13.7 \%$ \\
$\mathrm{TR}$ & - & - \\
\hline
\end{tabular}

\subsection{Application of the Models for $P_{\text {out }}$}

In (5.4) and (5.7), it was demonstrated how the predicted values of the model $\widehat{P}_{\text {out }}$ are found. The properties of approximation of the first- and second-order models can be easily examined using graphs.

For example, for the piecewise linear model (5.1)-(5.2), the graphs presented in Figure 3 show the local relationships between individual predictors and the dependent variable $\widehat{P}_{\text {out }}$. Figure 3(a) indicates that the pressure of the buffer gas (neon) $P_{\mathrm{NE}}$, which makes the biggest contribution to the model, should be taken within the interval $(16,21)$ Torr. For the inner diameter of the laser tube, the high values are achieved at $4<D<10 \mathrm{~mm}$ (Figure 3(b)), which has been established experimentally in $[16,17]$. The behavior of the pressure of hydrogen admixtures $P_{\mathrm{H} 2}$ also fits quite well for all experiments, exhibiting maximum local influence in [0.02, 0.04] Torr (Figure 3(e)). 
Table 4: Calculated values for predicted future experiments using MARS model (5.5)-(5.6).

\begin{tabular}{lcc}
\hline & $P_{\mathrm{IN}}, \mathrm{W}$ & $P_{\text {out }}, \mathrm{mW}$-predicted value \\
\hline$\# 1$ & 1500 & 1021 \\
$\# 2$ & 1550 & 1083 \\
$\# 3$ & 1600 & 1145 \\
$\# 4$ & 1650 & 1208 \\
$\# 5$ & 1700 & 1270 \\
\hline
\end{tabular}

Analogically, for the nonlinear model (5.5)-(5.6), Figures 4(a)-4(d) show local relationships between the main pairs of significant input parameters in two-dimensional regions. The supplied electrical power $P_{\mathrm{IN}}$ makes the biggest contribution to the model and shows an increasing behavior by comparing Figures $4(\mathrm{a}), 4(\mathrm{c})$ and $4(\mathrm{~d})$. Figures $4(\mathrm{~b})$ and $4(\mathrm{c})$, indicate that the neon pressure $P_{\mathrm{NE}}$ should be taken within the interval $(15,25)$ Torr. The hydrogen pressure gives an optimal contribution for $[0.03,0.04]$ Torr, according to Figures $4(\mathrm{~d})$ and $4(b)$.

In addition to the mutual influence of predictors, it is also possible to plot slices in order to examine the local behavior of each of the two variables with one fixed at a value chosen by the researcher and the other changing within the whole interval of definition.

As an example, Figure 5(a) shows a slice from Figure 4(b) for the modeled behavior of the variable $P_{\mathrm{NE}}$ (neon gas pressure) for a fixed value of the hydrogen pressure $P_{\mathrm{H} 2}=$ 0.03 Torr. In this case, there is a clearly identifiable maximum in the interval $[11,26]$ Torr. The slice in Figure 5(b) shows the distribution of the influence of the pair of predictors $\left\{P_{\mathrm{NE}}, P_{\mathrm{IN} 2}\right\}$ for a fixed $P_{\mathrm{NE}}=19.5$ Torr, depending on the increase of $P_{\mathrm{IN} 2}$, as an element of Figure 4 (c), which shows the maximum importance.

By carefully studying the resulting estimates of $\widehat{P}_{\text {out }}$ from a given model and the defined influences of the significant variables, as well as their interactions, it is possible to guide the experiment in order to improve the output characteristics.

In addition to the plots, the models can be used to examine the local influence of the main laser input quantities on output ones, as well as to predict new experiments. In order to demonstrate this, we will consider the local behavior of $P_{\text {out }}$ for the laser from [18] when varying the pressure of the neon buffer gas $P_{\mathrm{NE}}$ in the interval $[15.5,18.5]$ Torr. For $P_{\mathrm{IN}}=$ $1300 \mathrm{~W}$ we choose experiment data with fixed $D=\mathrm{DR}=7.1 \mathrm{~mm}, L=86.5 \mathrm{~cm}, P_{\mathrm{H} 2}=0.03$ Torr, $P_{\mathrm{RF}}=19.5 \mathrm{kHz}$, and $\mathrm{C}=735 \mathrm{pF}$. In Figure 6(a) continuous line indicates the experiment data, Predicted shows estimates from model $(40 ; 2)$.

Results for a future experiment prediction using model $(40 ; 2)$ are given in Table 4. The following are fixed: $D=\mathrm{DR}=7.1 \mathrm{~mm}, L=86.5 \mathrm{~cm}, P_{\mathrm{NE}}=16.7$ Torr, $P_{\mathrm{H} 2}=0.03$ Torr, $P_{\mathrm{RF}}=25 \mathrm{kHz}, \mathrm{TR}=490^{\circ} \mathrm{C}$, and $\mathrm{C}=372 \mathrm{pF}$. As expected, laser generation $P_{\text {out }}$ increases when input electric power $P_{\mathrm{IN}}$ is increased. In this table, the middle row \#3 corresponds to an actual experiment with the measured value of $P_{\text {out }}=1200 \mathrm{~mW}$, and the one predicted by the model is $1145 \mathrm{~mW}$, which represents a relative error of under $5 \%$.

\section{MARS Models for Laser Service Life}

This type of research has not been performed for metal vapor lasers so far. The models utilize as predictors all of the 9 input laser characteristics from Section 3 with response $L_{\text {Time }}$-laser service life, measured in hours. Again, our objective is to find a model with a sufficiently high 




(a)

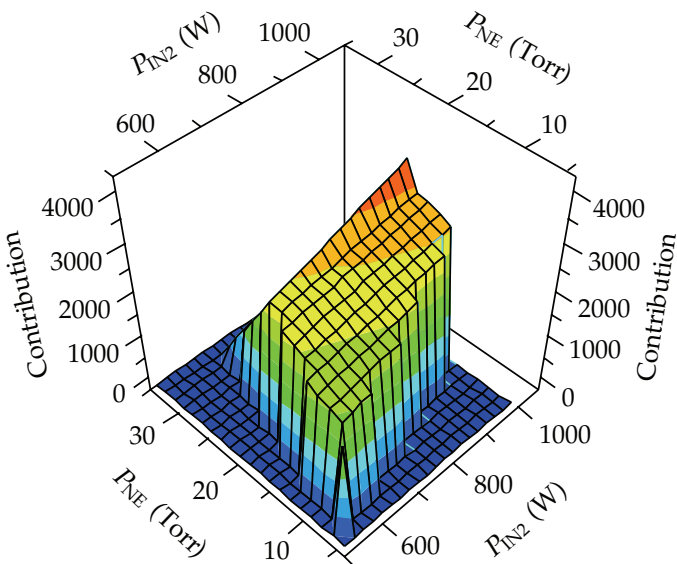

(c)

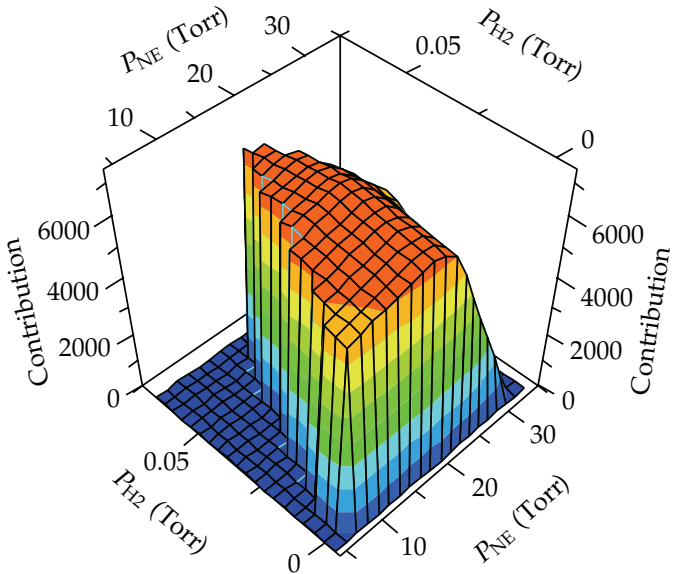

(b)

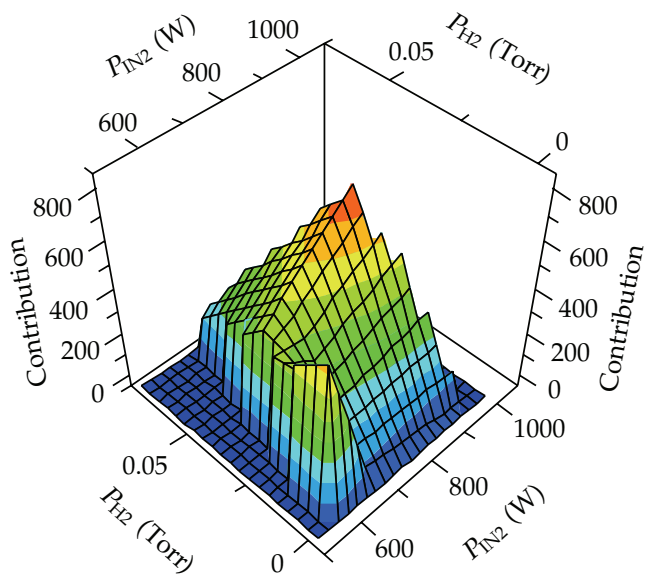

(d)

Figure 4: Graphs of contribution of the selected pairs of predictor variables in MARS model with (5.5)-(5.6) in ordinal units.

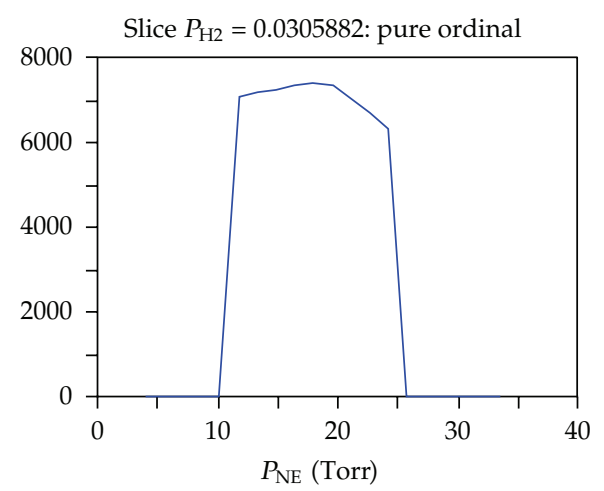

(a)

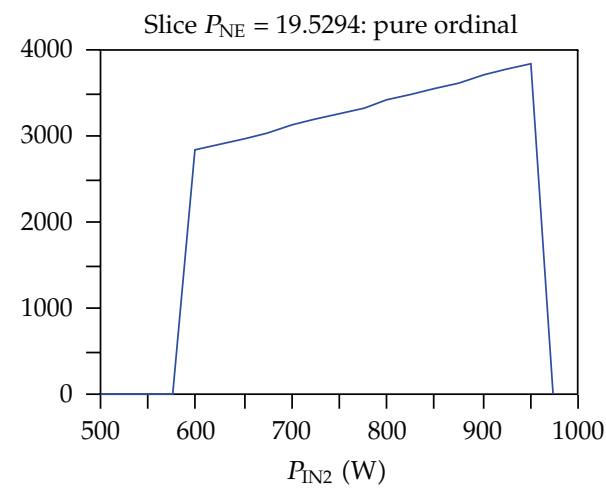

(b)

Figure 5: View of slices from Figures 4(b) and 4(c) for the influence of $P_{\mathrm{NE}}$ and $P_{\mathrm{IN} 2}$ on $P_{\text {out }}$ in pure ordinal units. 


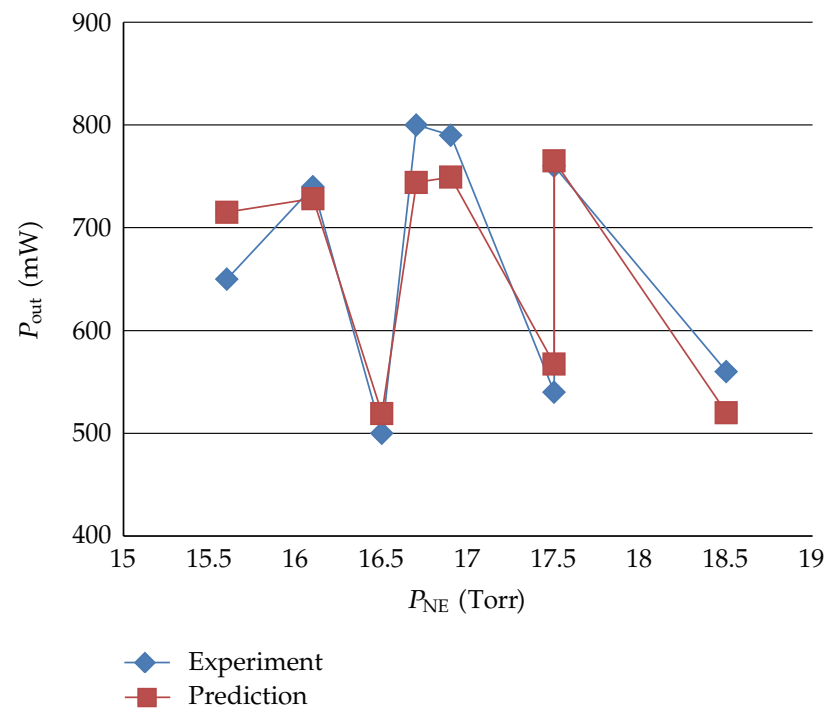

Figure 6: Comparison of output power $P_{\mathrm{out}}$ with values predicted by model $(40 ; 2)$ at varying $P_{\mathrm{NE}}$ for $P_{\mathrm{IN}}=$ $1300 \mathrm{~W}$

coefficient of determination (over 95\%) and the smallest possible mean-square error (under $5 \%$ ) comparable with the experimental error.

The statistics of the obtained models and basic statistics are given in Table 5. The first best MARS model with $98 \%$ coefficient of determination is model $(40 ; 2)$.

\subsection{Linear MARS Models of $L_{\text {Time }}$}

Of the linear-type models, we will present model $(30 ; 1)$. The figures of other linear models are given in the upper part of Table 5.

The calculated best MARS model $(30 ; 1)$ includes the following 6 basis functions with 4 predictors:

$$
\begin{gathered}
\mathrm{BF} 1=\max (0, \mathrm{TR}-500), \\
\mathrm{BF} 3=\max (0, C-372.184), \\
\mathrm{BF} 5=\max \left(0,8.4-P_{\mathrm{NE}}\right), \\
\mathrm{BF} 6=\max (0, \mathrm{TR}-560), \\
\mathrm{BF} 9=\max \left(0,650-P_{\mathrm{IN} 2}\right), \\
\mathrm{BF} 13=\max (0, \mathrm{TR}-550) .
\end{gathered}
$$

The regression model for laser service life $L_{\text {Time }}$ with these functions is

$$
\begin{aligned}
\widehat{L}_{\text {Time }}= & 693.836-12.8726 \mathrm{BF} 1-1.47903 \mathrm{BF} 3-397.699 \mathrm{BF} 5 \\
& +18.566 \mathrm{BF} 6+3.7592 \mathrm{BF} 9+9.86482 \mathrm{BF} 13 .
\end{aligned}
$$


Table 5: Main statistics of the constructed best MARS models of laser service life $L_{\text {Time }}$ of UV copper ion lasers.

\begin{tabular}{cccccc}
\hline Model & $R^{2}$ & MARS GCV $R^{2}$ & Std. Err. of the estimate & BF in model & Predictors \\
\hline $\mathbf{( 3 0 ; 1 )}$ & $\mathbf{0 . 9 9 1}$ & $\mathbf{0 . 9 8 9}$ & $\mathbf{2 2 . 5 3}$ & $\mathbf{6}$ & $\mathrm{TR}, \boldsymbol{C}, \boldsymbol{P}_{\mathrm{NE}}, \boldsymbol{P}_{\mathrm{IN} 2}$ \\
$(40 ; 1)$ & 0.991 & 0.989 & 22.55 & 6 & $\mathrm{TR}, \mathrm{C}, P_{\mathrm{NE}}, P_{\mathrm{IN} 2}, L$ \\
$(50 ; 1)$ & 0.991 & 0.989 & 22.53 & 6 & $\mathrm{TR}, C, P_{\mathrm{NE}}, P_{\mathrm{IN} 2}$ \\
\hline $\mathbf{( 3 0 ; 2 )}$ & $\mathbf{0 . 9 9 7}$ & $\mathbf{0 . 9 9 5}$ & $\mathbf{1 3 . 9 0}$ & $\mathbf{1 3}$ & $\mathrm{TR}, \boldsymbol{P}_{\mathrm{NE}}, \boldsymbol{C}, \boldsymbol{P}_{\mathrm{RF}}, \mathrm{DR}, \boldsymbol{P}_{\mathrm{IN} 2}$ \\
$(35 ; 2)$ & 0.997 & 0.995 & 13.90 & 13 & $\mathrm{TR}, P_{\mathrm{NE}}, C, P_{\mathrm{RF}}, \mathrm{DR}, P_{\mathrm{IN} 2}$ \\
$(40 ; 2)$ & 0.997 & 0.995 & 13.90 & 13 & $\mathrm{TR}, P_{\mathrm{NE}}, \mathrm{C}, P_{\mathrm{RF}}, \mathrm{DR}, P_{\mathrm{IN} 2}$ \\
\hline$(30 ; 3)$ & 1.000 & 0.999 & 0.14 & 21 & $\mathrm{TR}, C, P_{\mathrm{NE}}, \mathrm{DR}, P_{\mathrm{H} 2}$ \\
$(35 ; 3)$ & 1.000 & 0.999 & 0.33 & 24 & $\mathrm{TR}, \mathrm{C}, P_{\mathrm{NE}}, \mathrm{DR}, P_{\mathrm{H} 2}$ \\
$(40 ; 3)$ & 1.000 & 0.999 & 0.33 & 24 & $\mathrm{TR}, C, P_{\mathrm{NE}}, \mathrm{DR}, P_{\mathrm{H} 2}$ \\
\hline
\end{tabular}

Model (6.1)-(6.2) can be used to estimate and predict the service life of the examined lasers as shown by the models of $P_{\text {out }}$. The model is significant at level 0.000 .

The relative influence of individual predictors in model (6.2) is given in Table 6, column 2. It is apparent that the most significant variables are TR, C, $P_{\mathrm{NE}}$, and $P_{\mathrm{IN} 2}$ (in descending order according to their influence). The behavior of the 4 main predictors in their intervals of determination and contribution in model (6.1)-(6.2) are shown in Figures 7(a)$7(d)$ in pure ordinal units.

\subsection{Nonlinear MARS Models of $L_{T i m e}$}

Of the second-order models we present best model with up to $30 \mathrm{BFs}$, which is denoted as model $(30 ; 2)$. The obtained model includes the following 16 BFs:

$$
\begin{gathered}
\mathrm{BF} 1=\max (0, \mathrm{TR}-500), \\
\mathrm{BF} 3=\max (0, C-372.184), \\
\mathrm{BF} 4=\max \left(0, P_{\mathrm{NE}}-8.4\right), \\
\mathrm{BF} 5=\max \left(0,8.4-P_{\mathrm{NE}}\right), \\
\mathrm{BF} 6=\max (0, \mathrm{TR}-550) \mathrm{BF} 4, \\
\mathrm{BF} 7=\max (0,550-\mathrm{TR}) \mathrm{BF} 4, \\
\mathrm{BF} 8=\max \left(0, P_{\mathrm{NE}}-5.5\right) \mathrm{BF} 1, \\
\mathrm{BF} 10=\max (0,560-\mathrm{TR}), \\
\mathrm{BF} 12=\max (0,7.3-\mathrm{DR}) \mathrm{BF} 10, \\
\mathrm{BF} 13=\max \left(0, P_{\mathrm{NE}}-16.7\right) \mathrm{BF} 10, \\
\mathrm{BF} 15=\max \left(0, P_{\mathrm{NE}}-16.5\right) \mathrm{BF} 10, \\
\mathrm{BF} 17=\max \left(0, P_{\mathrm{IN} 2}-525\right) \mathrm{BF} 3, \\
\mathrm{BF} 18=\max (0, \mathrm{TR}-490) \mathrm{BF} 5,
\end{gathered}
$$




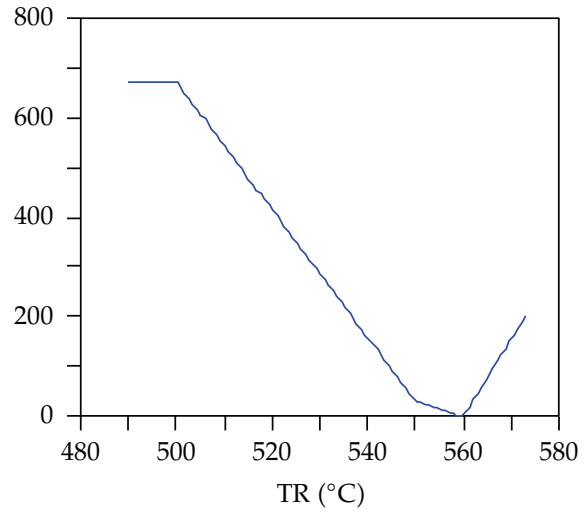

(a)

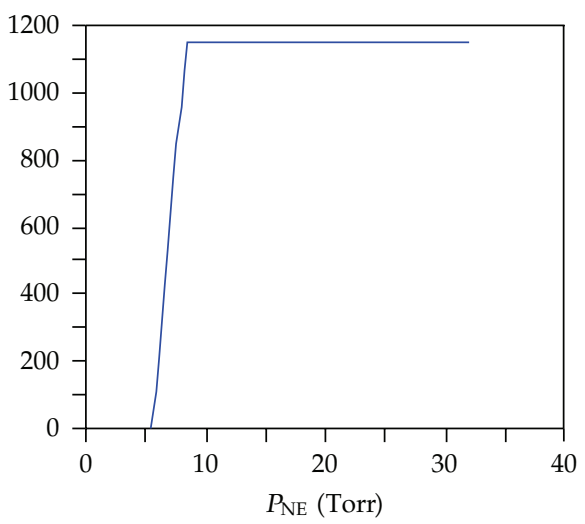

(c)



(b)

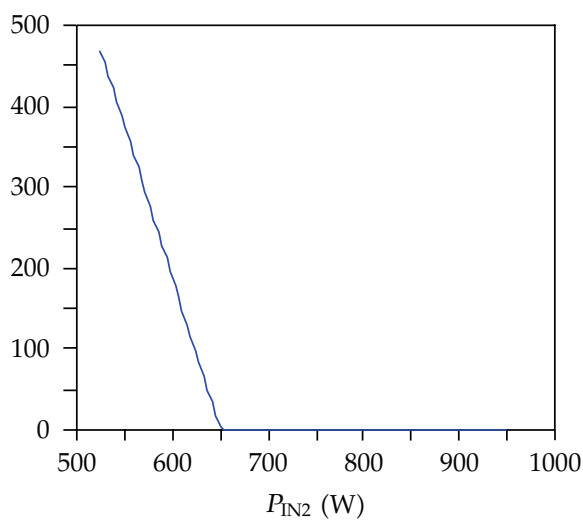

(d)

Figure 7: Graphs of the main predictors in model (30;1) with (6.1)-(6.2) showing their relationship with the response $L_{\text {Time }}$.

$$
\begin{aligned}
& \mathrm{BF} 21=\max \left(0, P_{\mathrm{IN} 2}-700\right) \mathrm{BF} 10, \\
& \mathrm{BF} 23=\max \left(0, P_{\mathrm{IN} 2}-750\right) \mathrm{BF} 10, \\
& \mathrm{BF} 25=\max \left(0, P_{\mathrm{RF}}-19.5\right) \mathrm{BF} 1 .
\end{aligned}
$$

The corresponding regression model for laser service life $L_{\text {Time }}$ includes 13 of these functions and 6 predictors. It has the following form:

$$
\begin{aligned}
\widehat{L}_{\text {Time }}= & -103.913-2.59996 \mathrm{BF} 3-22.9161 \mathrm{BF} 4-2.00281 \mathrm{BF} 6+2.35338 \mathrm{BF} 7 \\
& +0.71495 \mathrm{BF} 8-4.50066 \mathrm{BF} 12+8.48605 \mathrm{BF} 13-9.19289 \mathrm{BF} 15 \\
& +0.0105753 \mathrm{BF} 17+0.456027 \mathrm{BF} 18-0.112225 \mathrm{BF} 21+0.104861 \mathrm{BF} 23 \\
& +1.12861 \mathrm{BF} 25 .
\end{aligned}
$$

The absolute error of model (6.3)-(6.4) is about $0.006 \%$, and the relative one is $4 \%$. The importance of the two main predictors is shown in Figures $8(a)-8(b)$. 
Table 6: Relative variable importance in the considered MARS models (30;1), described by (6.1)-(6.2), and (30;2) with (6.3)-(6.4), for laser service life $L_{\text {Time }}$ of UV copper ion laser.

\begin{tabular}{lcc}
\hline Variable & Model $(30 ; 1)$ & Model $(30 ; 2)$ \\
\hline TR & $100 \%$ & $100 \%$ \\
$P_{\mathrm{NE}}$ & $20.1 \%$ & $39.2 \%$ \\
$C$ & $46.7 \%$ & $30.8 \%$ \\
$P_{\mathrm{RF}}$ & - & $2.5 \%$ \\
$\mathrm{DR}$ & - & $2 \%$ \\
$P_{\mathrm{IN} 2}$ & $10.8 \%$ & $1.6 \%$ \\
\hline
\end{tabular}

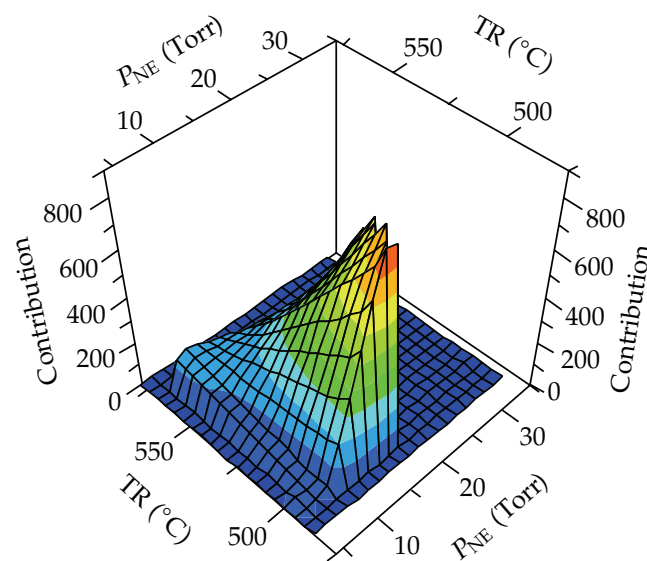

(a)

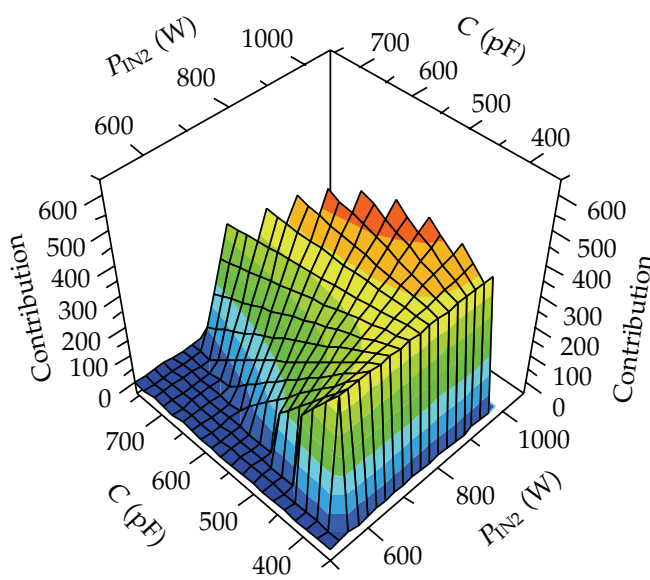

(b)

Figure 8: Graphs of the local mutual contribution of the main predictors to model $(30 ; 2)$.

\subsection{Application of the Obtained MARS Models of $L_{\text {Time }}$}

Model (6.3)-(6.4) analogically allows for estimation and prediction of the service life of the considered laser device depending on a given set of values of participating predictors. What is essential here is the relative influence of these predictors, which is to be taken into consideration by the design engineer when planning the experiment, in our case maintaining strictly the temperature of the reservoirs TR (equal to the temperature maintained at the outer wall of the laser tube (quartz or ceramic), and keeping the ratio between the pressure of the neon buffer gas $P_{\mathrm{NE}}$ and the bank of condenser within the established limits.

\section{Comparison and Diagnosis of the Obtained MARS Models}

Model comparison can be performed using the general statistical indices from Tables 2 and 5. It has to be noted that in the last column the predictors for the respective model are presented in descending order according to their relative importance.

The lowest values of $R^{2}$ and GCV $R^{2}$ are in linear models $(r=1)$ both for $P_{\text {out }}$ and for $L_{\text {Time. }}$. With the increase of the number of basis functions $(B F s)$ the parameters of the models increase slightly. However, the investigation of the residuals of the linear models shows that 
they are not sufficiently adequate and that the relative error is comparatively high within 10$15 \%$. This means that the relationship of the input independent variables with $P_{\text {out }}$ or $L_{\text {Time }}$ is not linear.

For output power $P_{\text {out }}$, Table 2 shows that second-order models from $(40 ; 2)$ exhibit a $98 \%$ coefficient of determination and that the participation of the predictors is stable. Since for $(40 ; 2)$ the GCV $R^{2}$ estimate is over $95 \%$, this model is quite good. The next models (up to $(60 ; 2))$ demonstrate practically the same fitting properties.

Of all models of $L_{\text {Time, }}$ model $(30 ; 2)$ exhibits the best qualities.

Third-order models are comparable with the respective second-order models and demonstrate almost the same or slightly lower indices than second-order ones. Since these models are more complex in form, we conclude that the examined data demonstrate second degree local nonlinearities and it is best to describe these using second-order models.

In order to examine the diagnostics we have to note that all presented models are statistically significant at level $P=0.00000$. The coefficients of the models are significant with $P \leq 0.005$. The standardized error of the estimate is small. Model residuals are normally distributed.

Figure 9 shows the comparison of experimental data for laser output power $P_{\text {out }}$ with those calculated by model $(40 ; 2)$. It is observed that the model fits data quite well. For the coefficient of determination we have $R^{2}=0.98$, which means that the model accounts for $98 \%$ of all data. The corresponding GCV $R^{2}=0.95$ (see Table 2). The residuals of this model are normally distributed with $N(0,0.998)$.

This way, the two main criteria and statistical indices show very good predictive properties and goodness of fit of the constructed MARS model $(40 ; 2)$. Figure 10.

The fitting property of model $(30 ; 2)$ for $L_{\text {Time }}$ against the experiment is given in

\section{Physical Interpretation of the Results}

The developed models correspond quite well with the experiment and reflect nonlinear local relationships in a multidimensional space of 9 variables.

Laser output power is most significantly influenced by input electric power, neon pressure, and hydrogen pressure. Of the geometric dimensions, output power is most dependent on the diameter of the rings DR, which for latest laser devices is equal to $D$ - the diameter of the inner tube, with its optimal value between 7.1 and $7.3 \mathrm{~mm}$.

The new results are those for the service life of the examined lasers, which is one of the most important issues for all types of devices. For the latest lasers [20] this period is quite acceptable, reaching 700-1000 hours. Furthermore, periodically a refreshment of laser tube is made, which involves shutting down the laser, cleaning, and changing the gas mixture. The obtained models unequivocally show that the main contribution to extending the service life of the laser is that of TR (inversely proportional-Figure 7(a)), C, and $P_{\mathrm{NE}}$. Therefore, these characteristics need to be considered carefully when designing new lasers of the investigated type and their parameters should be constant or nearly constant in relation to those already established.

\section{Conclusion}

The basic results of the performed statistical modeling of laser output characteristics, laser generation and laser service life of a multiline ultraviolet copper ion vapor laser excited in a 


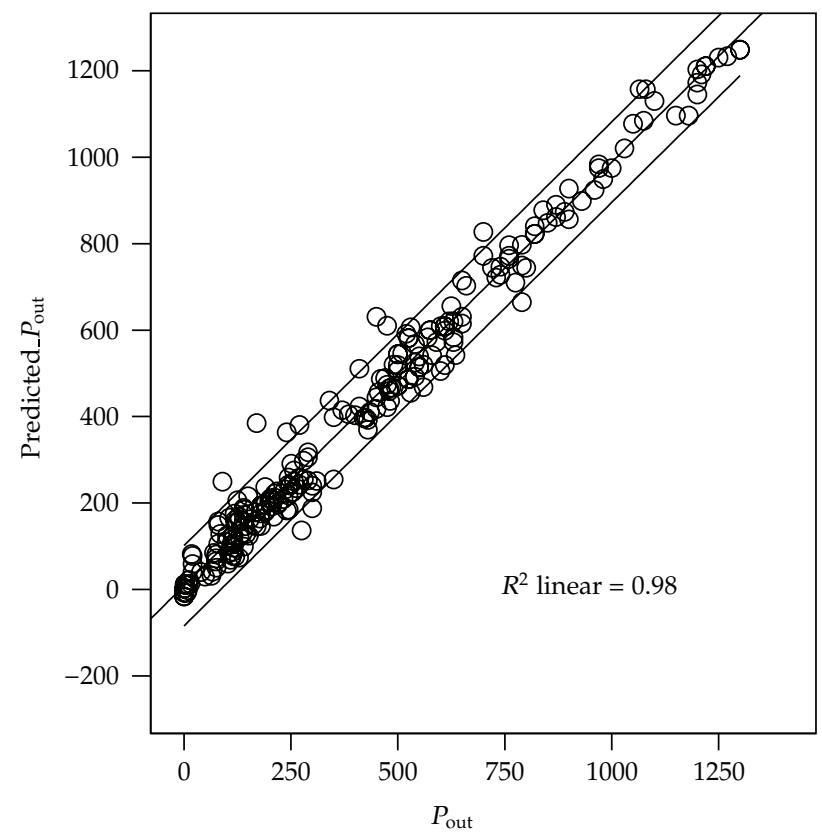

Figure 9: Values of the experimental $P_{\text {out }}$ against the predicted $\widehat{P}_{\text {out }}$ by model $(40 ; 2)$ with (5.5)-(5.6) with $5 \%$ confidential interval.

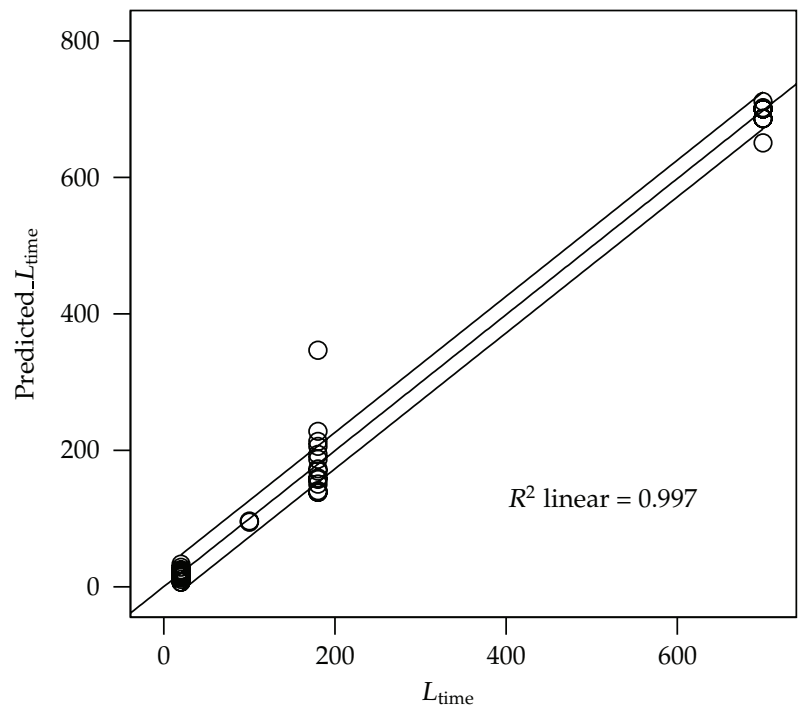

Figure 10: Values of the experimental $L_{\text {Time }}$ against the predicted $\widehat{L}_{\text {Time }}$ by model $(30 ; 2)$ with $(6.3)-(6.4)$ with $5 \%$ confidential interval.

longitudinal pulsed discharge, have been obtained using nonparametric MARS models. The models demonstrate very good abilities and goodness of fit when predicting existing and future experiments.

It was determined that the best MARS models are nonlinear and contain secondorder members. Of 9 input independent variables used as predictors, 8 influence laser output 
power with the most significant ones being the pressure of the applied electric power, the inner diameter of the laser tube (diameter of the rings), neon buffer gas pressure, and hydrogen pressure. Service life models are simpler and only 3 laser characteristics exhibit significant influence: temperature of the tube, neon gas pressure, and equivalent capacity of the condensation battery.

It is shown that the models can be used to estimate and predict local properties of laser generation and service life of the devices.

The techniques developed can also be further employed in the process of future industrial application of this type of lasers.

\section{Acknowledgments}

This paper is published in cooperation with Project of the Bulgarian Ministry of Education, Youth and Science, BG051PO001-3.3-05/0001 "Science and Business," financed under Operational program "Human Resources Development" by the European Social Fund.

\section{References}

[1] M. J. Kushner and B. E. Warner, "Large-bore copper-vapor lasers: kinetics and scaling issues," Journal of Applied Physics, vol. 54, no. 6, pp. 2970-2982, 1983.

[2] "Numerical modeling of low-temperature plasmas," in Encyclopedia of Low-temperature Plasma, Series B, M. Ianus, Ed., vol. 7, Moscow, Russia, 2004.

[3] A. M. Boichenko, G. S. Evtushenko, and S. N. Torgaev, "Simulation of a CuBr laser," Laser Physics, vol. 18, no. 12, pp. 1522-1525, 2008.

[4] B. L. Pan, G. Chen, B. N. Mao, and Z. X. Yao, "Kinetic process of UV Cu+ laser in Ne-CuBr longitudinal pulsed discharge," Optics Express, vol. 14, no. 19, pp. 8644-8653, 2006.

[5] M. Bang-Ning, P. Bai-Liang, C. Li, W. Ya-Juan, and Y. Zhi-Xin, "Kinetic analysis of the factors limiting the output power of the Ne-CuBr UV laser," Chinese Physics B, vol. 18, no. 4, pp. 1542-1546, 2009.

[6] S. G. Gocheva-Ilieva and I. P. Iliev, Statistical Models of Characteristics of Metal Vapor Lasers, Nova Science Publishers, New York, NY, USA, 2011.

[7] I. P. Iliev, S. G. Gocheva-Ilieva, D. N. Astadjov, N. P. Denev, and N. V. Sabotinov, "Statistical analysis of the CuBr laser efficiency improvement," Optics and Laser Technology, vol. 40, no. 4, pp. 641-646, 2008.

[8] I. P. Iliev, S. G. Gocheva-Ilieva, D. N. Astadjov, N. P. Denev, and N. V. Sabotinov, "Statistical approach in planning experiments with a copper bromide vapor laser," Quantum Electronics, vol. 38, no. 5, pp. 436-440, 2008.

[9] S. G. Gocheva-Ilieva and I. P. Iliev, "Parametric and nonparametric empirical regression models: case study of copper bromide laser generation," Mathematical Problems in Engineering, vol. 2010, Article ID 697687, 15 pages, 2010.

[10] S. G. Gocheva-Ilieva and I. P. Iliev, "Modeling and prediction of laser generation in UV copper bromide laser via MARS," in Proceedings of the 5th International Conference on Optics, Astrophysics and Astronomy (ICOAA '10), O. Martin, Ed., Advanced Research in Physics and Engineering Series, Mathematics and Computers in Science and Engineering, pp. 166-171, WSEAS Press, Cambridge, UK, 2010.

[11] N. Vuchkov, N. Sabotinov, and K. Temelkov, “Ultraviolet Copper laser,” Patent Reg. no. 103312, 06.04.1999, Published Applications for Inventions, BG 10/2000.

[12] N. K. Vuchkov, K. A. Temelkov, P. V. Zahariev, and N. V. Sabotinov, "Laser tube for ultraviolet copper laser," Patent reg., no. 106759, 30.05.2002, Published Applications for Inventions BG 11/2003.

[13] N. K. Vuchkov, K. A. Temelkov, P. V. Zahariev, and P. V. N. V, "Laser tube for ultraviolet copper laser," Bulgaria Patent No.64880, 03.08.2006.

[14] N. K. Vuchkov, K. A. Temelkov, and N. V. Sabotinov, “UV lasing on Cu+ in a Ne-CuBr pulsed longitudinal discharge," IEEE Journal of Quantum Electronics, vol. 35, no. 12, pp. 1799-1804, 1999.

[15] N. K. Vuchkov, K. A. Temelkov, P. V. Zahariev, and N. V. Sabotinov, “Optimization of a UV Cu laser excited by pulse-longitudinal $\mathrm{Ne}-\mathrm{CuBr}$ discharge," IEEE Journal of Quantum Electronics, vol. 37, no. 4, pp. 511-517, 2001. 
[16] N. K. Vuchkov, K. A. Temelkov, P. V. Zahariev, and N. V. Sabotinov, "Influence of the active zone diameter on the UV-ion Ne-CuBr laser performance," IEEE Journal of Quantum Electronics, vol. 37, no. 12, pp. 1538-1546, 2001.

[17] N. K. Vuchkov, K. A. Temelkov, P. V. Zahariev, and N. V. Sabotinov, “Output parameters and a spectral study of UV Cu+ Ne-CuBr laser," Optics and Laser Technology, vol. 36, no. 1, pp. 19-25, 2004.

[18] N. K. Vuchkov, K. A. Temelkov, P. V. Zahariev, and N. V. Sabotinov, "A new laser tube construction for the UV Cu+ Ne-CuBr laser," in Laser and Laser Information Technologies, pp. 173-176, bgr, October 2003.

[19] N. K. Vuchkov, K. A. Temelkov, and N. V. Sabotinov, "Effect of hydrogen on the average output power of the UV Cu+ Ne-CuBr laser," IEEE Journal of Quantum Electronics, vol. 41, no. 1, pp. 62-65, 2005.

[20] N. Vuchkov, "High discharge tube resource of the UV Cu+ Ne-CuBr laser and some applications," in New Development in Lasers and Electric-Optics Research, pp. 41-74, Nova Science Publishers, New York, NY, USA, 2007.

[21] D. Steinberg, B. Bernstein, P. Colla, and K. Martin, MARS User Guide, Salford Systems, San Diego, Calif, USA, 2001

[22] 2012, http://www.salfordsystems.com/mars.php.

[23] M. Ilieva, V. Tsakova, N. K. Vuchko, K. A. Temelkov, and N. V. Sabotinov, "UV copper ion laser treatment of poly(3,4-ethylenedioxythiophene)," Journal of Optoelectronics and Advanced Materials, vol. 9, no. 2, pp. 303-306, 2007.

[24] M. Ilieva, A. Stoyanova, V. Tsakova et al., "Effect of deep UV laser treatment on electroless silver precipitation in supported poly-3,4-ethylenedioxythiophene layers," Journal of Optoelectronics and Advanced Materials, vol. 11, no. 10, pp. 1444-1447, 2009.

[25] J. Mouchovski, K. Temelkov, N. Vuchkov, and N. Sabotinov, "Calcium strontium fluoride crystals with different composition for UV-Laser application. Control of the growing rate and optical properties," Comptes Rendus de L'Academie Bulgare des Sciences, vol. 62, no. 6, pp. 687-694, 2009.

[26] J. H. Friedman, "Multivariate adaptive regression splines," The Annals of Statistics, vol. 19, no. 1, pp. 1-141, 1991. 


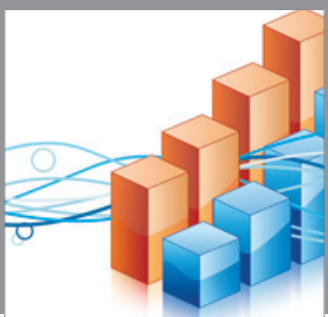

Advances in

Operations Research



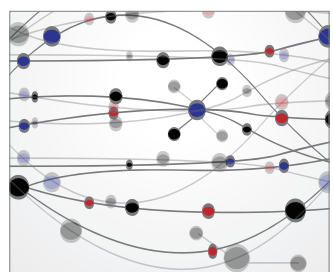

\section{The Scientific} World Journal


International Journal of

Mathematics and

Mathematical

Sciences
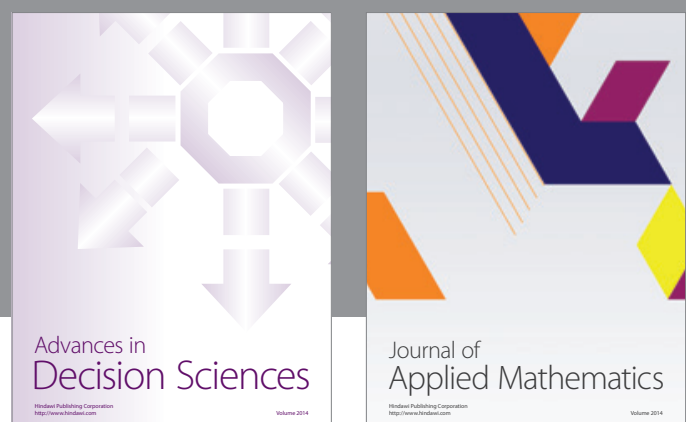

Journal of

Applied Mathematics
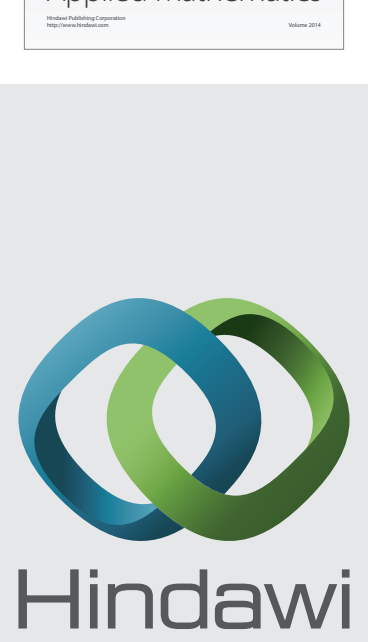

Submit your manuscripts at http://www.hindawi.com
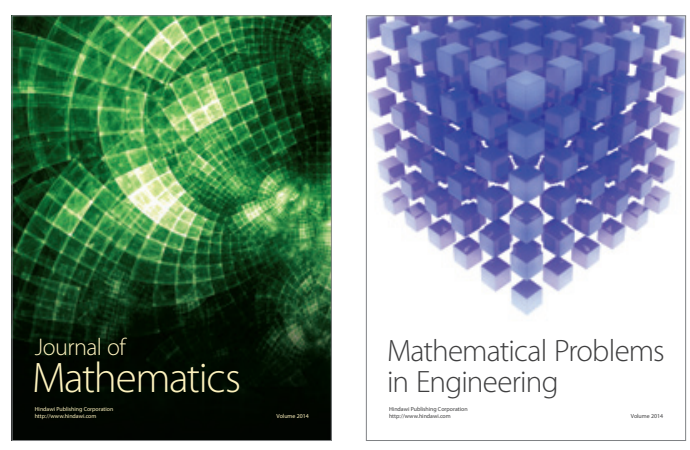

Mathematical Problems in Engineering
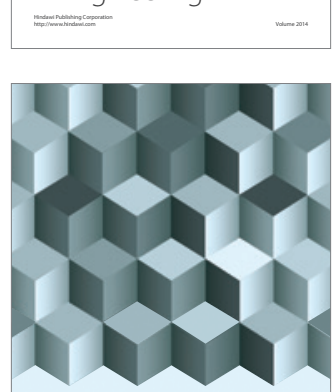

Journal of

Function Spaces
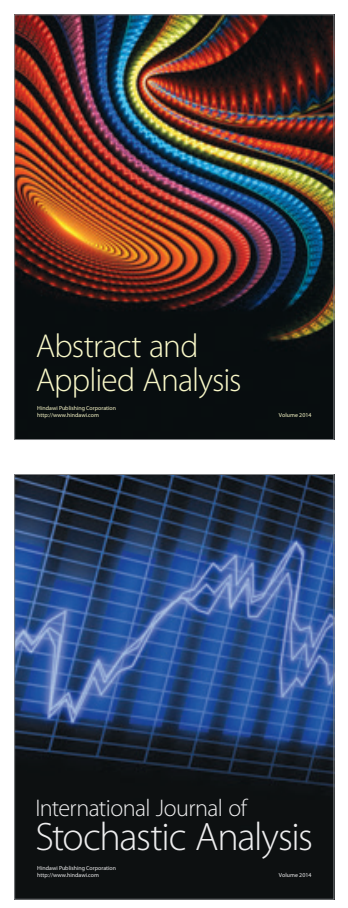

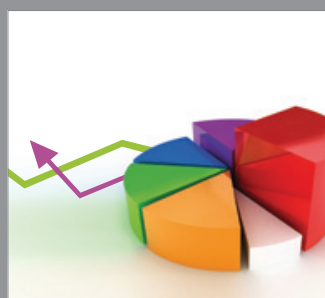

ournal of

Probability and Statistics

Promensencen
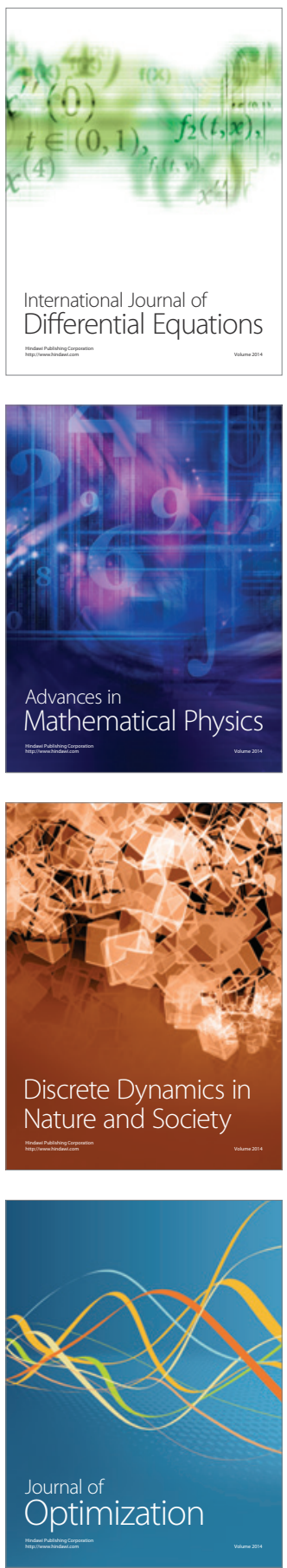